\title{
Effects of sedation during upper gastrointestinal endoscopy on endocrine response and cardiorespiratory function
}

G. Yetkin ${ }^{1}$, S. Oba ${ }^{2}$, M. Uludag1, I. Paksoy², I. Akgün ${ }^{1}$ and N. Eren ${ }^{3}$

\author{
${ }^{1}$ Department of Surgery and Surgical Endoscopy Unit, \\ ${ }^{2}$ Department of Anesthesiology, ${ }^{3}$ Department of Biochemistry, \\ Şişli Etfal Training and Research Hospital, Istanbul, Turkey
}

Correspondence

G. Yetkin

Ataköy 3. kisim O/8 blok. Daire

7 Bakirköy

Istanbul 34158

Turkey

E-mail: dryetkin@yahoo.com

GSM: 05326139471

Received January 31, 2007

Accepted July 31, 2007

\begin{abstract}
Upper gastrointestinal endoscopy is often accompanied by tachycardia which is known to be an important pathogenic factor in the development of myocardial ischemia. The pathogenesis of tachycardia is unknown but the condition is thought to be due to the endocrine response to endoscopy. The purpose of the present study was to investigate the effects of sedation on the endocrine response and cardiorespiratory function. Forty patients scheduled for diagnostic upper gastrointestinal endoscopy were randomized into 2 groups. While the patients in the first group did not receive sedation during upper gastrointestinal endoscopy, the patients in the second group were sedated with intravenous midazolam at the dose of $5 \mathrm{mg}$ for those under 65 years or $2.5 \mathrm{mg}$ for those aged 65 years or more. Midazolam was administered by slow infusion. In both groups, blood pressure, ECG tracing, heart rate, and peripheral oxygen saturation $\left(\mathrm{SpO}_{2}\right)$ were monitored during endoscopy. In addition, blood samples for the determination of cortisol, glucose and C-reactive protein levels were obtained from patients in both groups prior to and following endoscopy. Heart rate and systolic arterial pressure changes were within normal limits in both groups. Comparison of the two groups regarding the values of these two parameters did not reveal a significant difference, while a statistically significant reduction in $\mathrm{SpO}_{2}$ was found in the sedation group. No significant differences in serum cortisol, glucose or C-reactive protein levels were observed between the sedated and non-sedated group. Sedation with midazolam did not reduce the endocrine response and the tachycardia developing during upper gastrointestinal endoscopy, but increased the reduction in $\mathrm{SpO}_{2}$.
\end{abstract}

\section{Introduction}

The value of diagnostic and therapeutic endoscopic applications in gastrointestinal diseases is increasing progressively. How-
Key words

- Stress response

- Oxygen saturation

- Sedation

- Upper gastrointestinal

endoscopy

- Tachycardia 
cardia during upper gastrointestinal endoscopy (1-3).

Although the use of sedation during upper gastrointestinal endoscopy is prevalent $(4,5)$, there is much debate about the administration of sedatives. While some investigators propose that sedation-related complications may develop $(6,7)$, many have reported that conscious sedation increases comfort during upper gastrointestinal endoscopy and reduces cardiac stress $(8,9)$.

In the present study, we investigated the effects of sedation on the hemodynamic parameters and classical endocrine stress response that may cause tachycardia during routine upper gastrointestinal endoscopy.

\section{Patients and Methods}

Forty volunteer patients with ASA scores of 1 or 2 , who were referred to the endoscopy laboratory for diagnostic upper gastrointestinal endoscopy were included in the study, which was approved by the local Ethics Committee. The patients gave written informed consent to participate and were randomized to two groups by means of sealed envelopes.

The two groups of patients of 20 each did not receive premedication prior to gastroscopy. Lidocaine spray (Xylocaine ${ }^{\circledR}$ pump spray, Astra Zeneca, Lund, Sweden) was used for topical pharyngeal anesthesia. None of the patients received supplementary oxy-

Table 1. Physical characteristics of the patients and duration of endoscopy.

\begin{tabular}{lcc}
\hline & $\begin{array}{c}\text { Group 1 } \\
(\mathrm{N}=20)\end{array}$ & $\begin{array}{c}\text { Group 2 } \\
(\mathrm{N}=20)\end{array}$ \\
\hline Age (years) & 43.85 & 46.35 \\
Sex ratio (male:female) & $11: 9$ & $12: 8$ \\
Mean endoscopy time (min) & 6.54 & 7.25 \\
\hline
\end{tabular}

Group 1: no sedation. Group 2: iv midazolam (<65 years old: $5 \mathrm{mg}$; $>65$ years old: $2.5 \mathrm{mg}$ ). There were no statistical differences between groups (Mann-Whitney U-test). gen during gastroscopy. All patients were investigated by the same endoscopist (G.Y.) using the same endoscope (Olympus GIF XQ 230 videoendoscope, $9.8 \mathrm{~mm}$ in diameter, Olympus Optical Co. Ltd., Tokyo, Japan). The patients in group 1 did not receive sedation, whereas the patients in group 2 were sedated with intravenous midazolam at the dose of $2.5 \mathrm{mg}$ for subjects aged 65 years or older, or at the dose of $5 \mathrm{mg}$ for subjects younger than 65 years. Midazolam was administered by slow infusion into the antecubital vein at a single dose.

Continuous monitoring of blood pressure, ECG tracing, heart rate, and peripheral oxygen saturation $\left(\mathrm{SpO}_{2}\right)$ was performed using a Nikon-Kohden BSM 4113-K (Tokyo, Japan) standard monitor in both groups. Values were recorded $10 \mathrm{~min}$ prior to, during, and $20 \mathrm{~min}$ following the endoscopic examination. Also, blood samples were obtained from both groups prior to and $10 \mathrm{~min}$ and $1 \mathrm{~h}$ after endoscopy for the determination of cortisol, glucose, and C-reactive protein (CRP).

Data were analyzed statistically by the Mann-Whitney U-test for comparisons between groups and by the Tukey test for comparisons within groups, with the level of significance set at $\mathrm{P}<0.05$.

\section{Results}

All 40 patients tolerated and successfully completed the procedure. The mean duration of endoscopy was $6.54 \mathrm{~min}$ (range: 4.57-10.38 $\mathrm{min}$ ) for group 1 (non-sedated) and $6.85 \mathrm{~min}$ (range: 4.38-9.9 $\mathrm{min}$ ) for group 2 (sedated), with no significant difference between groups ( $\mathrm{P}=0.4382)$. Mean age and sex distribution were similar in both groups (Table 1). The effects of sedation on the endocrine response and cardiorespiratory function prior to, during and following endoscopy are shown in Table 2.

The values of heart rate, systolic blood pressure, and $\mathrm{SpO}_{2}$ obtained for both groups 
are displayed in Figure 1A,B and C, respectively. Heart rate and systolic arterial pressure increased in both groups during the endoscopic procedure, but arrhythmias and signs of myocardial ischemia were not observed in any of the patients. The increases in heart rate during endoscopy were nonsignificant in both the non-sedated group and the sedated group. In addition, no statistically significant difference was found between groups regarding the simultaneous values of heart rate both during and after endoscopy. Similar results were also obtained in the statistical analysis of the alterations in systolic arterial pressure. The increments in systolic arterial pressure observed during endoscopy were nonsignificant in both group 1 and group 2. Compari- son of the simultaneous values of systolic blood pressure between groups also revealed no significant difference either during or following endoscopy.

$\mathrm{SpO}_{2}$ levels decreased during endoscopy in both groups, but while this reduction was nonsignificant in the group without sedation, a statistically significant reduction was found in the sedation group $(\mathrm{P}=0.002)$. In both groups, $\mathrm{SpO}_{2}$ levels increased to preendoscopy values following endoscopy, and comparison of the pre- and post-endoscopy $\mathrm{SpO}_{2}$ levels revealed no statistically significant difference in either group. Also, no statistically significant difference was found between groups regarding the simultaneous $\mathrm{SpO}_{2}$ levels during and following endoscopy.

\begin{tabular}{|c|c|c|c|c|c|c|}
\hline & $\begin{array}{l}\text { Glucose } \\
\text { (mg/dL) }\end{array}$ & $\begin{array}{l}\text { Cortisol } \\
(\mu \mathrm{g} / \mathrm{dL})\end{array}$ & $\begin{array}{l}\text { CRP } \\
(\mathrm{mg} / \mathrm{L})\end{array}$ & $\begin{array}{l}\text { Heart rate } \\
(\mathrm{bpm})\end{array}$ & $\begin{array}{l}\text { Blood pressure } \\
\qquad(\mathrm{mmHg})\end{array}$ & $\begin{array}{c}\mathrm{SpO}_{2} \\
(\%)\end{array}$ \\
\hline \multicolumn{7}{|c|}{ Before endoscopy } \\
\hline Group 1 & $80.2 \pm 12.24$ & $18.84 \pm 4.98$ & $3.1 \pm 1.98$ & $94.65 \pm 18.81$ & $140.7 \pm 31.79$ & $96.51 \pm 1.04$ \\
\hline Group 2 & $86.1 \pm 10.13$ & $15.9 \pm 4.12$ & $3.6 \pm 1.61$ & $93 \pm 13.43$ & $135.1 \pm 19.86$ & $96.9 \pm 1.23$ \\
\hline \multicolumn{7}{|c|}{ During endoscopy } \\
\hline Group 1 & $85.25 \pm 11.7$ & $19.03 \pm 4.72$ & $3.88 \pm 1.95$ & $98.5 \pm 15.73$ & $148.95 \pm 23.98$ & $95.7 \pm 1.52$ \\
\hline Group 2 & $90.25 \pm 9.49$ & $16.12 \pm 5.21$ & $3.71 \pm 1.7$ & $100.8 \pm 12.6$ & $138.3 \pm 27.41$ & $95.14 \pm 1.87$ \\
\hline \multicolumn{7}{|c|}{ After endoscopy } \\
\hline Group 1 & $90.7 \pm 19.73$ & $18.22 \pm 4.18$ & $3.74 \pm 2.08$ & $100.65 \pm 12.85$ & $133.95 \pm 23.71$ & $96.18 \pm 1.49$ \\
\hline Group 2 & $86.4 \pm 10.05$ & $16.75 \pm 2.55$ & $3.75 \pm 1.56$ & $94.1 \pm 15.19$ & $129.9 \pm 24.21$ & $95.85 \pm 1.54$ \\
\hline
\end{tabular}

Data are reported as mean \pm SD for 20 patients in each group. See Table 1 for definition of groups. CRP $=C$-reactive protein; $\mathrm{SpO}_{2}=$ peripheral oxygen saturation. There were no statistical differences between groups (Mann-Whitney Utest).
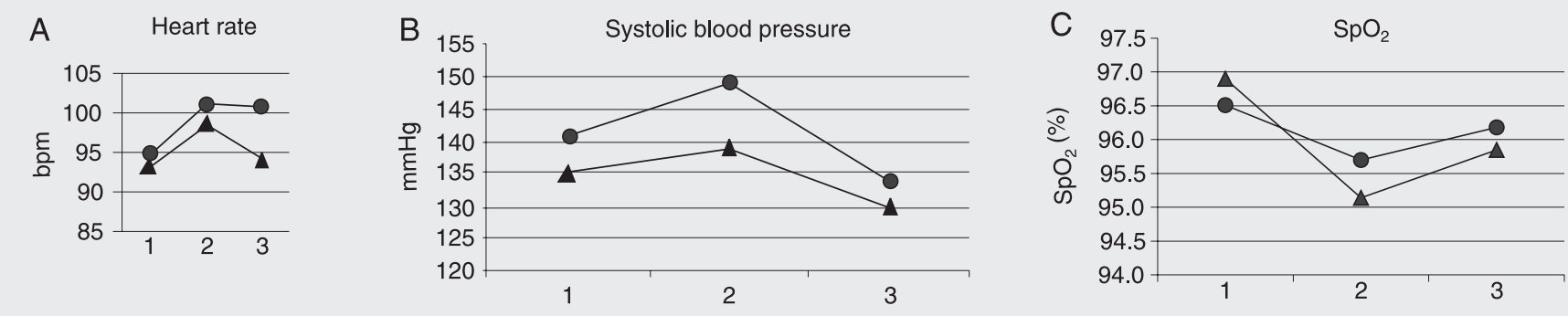

Figure 1. Changes in cardiopulmonary parameters (heart rate $(A)$, systolic blood pressure $(B)$, and peripheral oxygen saturation $\left(\mathrm{SpO}_{2}, \mathrm{C}\right)$ detected before (1), during (2), and after (3) endoscopy. Circles = non-sedated group; triangles = sedated group. 
Serum cortisol, glucose and CRP levels increased following endoscopy in both groups; however, none of the increments in these parameters was statistically significant. Also, comparison of the groups regarding the simultaneous levels of these parameters revealed no statistically significant difference (Figure 2A-C).

\section{Discussion}

An organism undergoing trauma initiates a systemic response against the trauma in order to maintain homeostasis. Secretion of the mediators, and the intracellular and intercellular metabolic alterations that initiate the neuroendocrine response developing after trauma depend on the duration and type of trauma $(10,11)$. Regardless of its duration, gastroscopic examination is also a trauma which elicits a metabolic endocrine response in the body. The temporary endocrine response occurring in the body during gastroscopic examination resembles the welldescribed classical stress response developing against surgery (12). Afferent vagal, sympathetic and phrenic mechanoreceptors exist in the esophagus and stomach. It has been proposed that mechanical autonomic stimulation of these organs during gastroscopy may initiate the endocrine response developing during endoscopy. The symptoms of intolerance to endoscopy such as hypoxia, pain and coughing that develop during the endoscopic procedure may cause a stronger response. The endocrine hormones that are secreted may play a role in the development of tachycardia which emerges during endoscopy, by affecting the target organs including the cardiovascular system $(13,14)$.

Tachycardia is known to be an important pathogenic factor in the development of myocardial ischemia that may occur during endoscopy (15). Therefore, it has been suggested that $B$-adrenergic blockade of the heart, which is one of the target organs of the endocrine stress, might prevent the development of myocardial ischemia, as observed in a study in which $B$-adrenergic blockade induced by metoprolol was shown to reduce the incidences of tachycardia and myocardial ischemia during endoscopy (16). Similar to the target organ blockade seen here, reduction of afferent stimulation has been proposed to be effective in reducing the stress response emerging during upper gastrointestinal endoscopy (12). Particularly, upper gastrointestinal endoscopy performed with induction of sedation has been reported to be more comfortable than endoscopy without sedation, and sedation has been proposed to reduce the stress response (8). Thus, in the present study, we investigated whether sedation reduces the stress response and tachycardia occurring in upper gastrointestinal endoscopy.

In several studies, the classical metabolic endocrine response has been obtained with

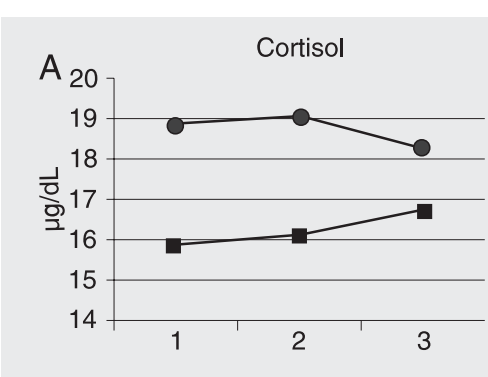

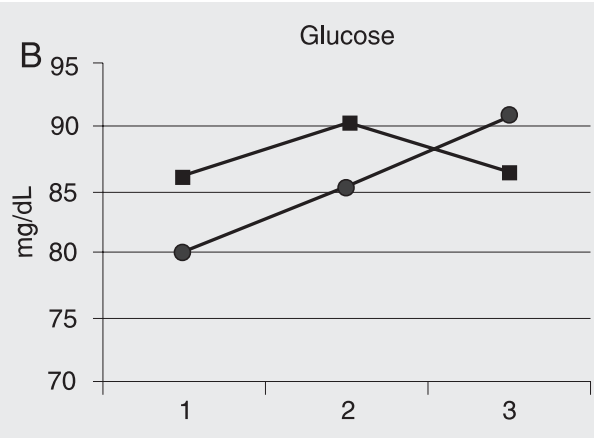

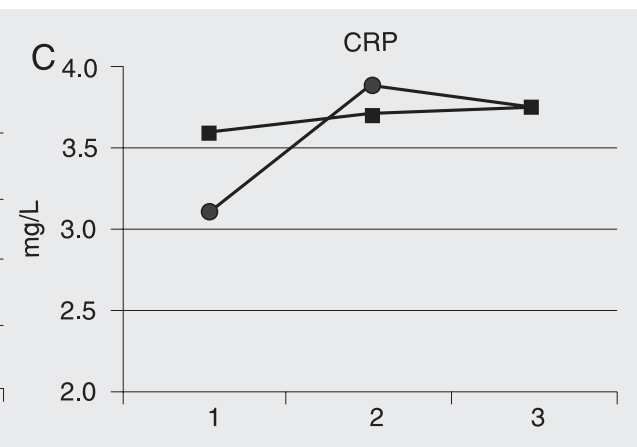

Figure 2. Serum cortisol (A), glucose (B), and C-reactive protein (CRP, C) concentrations detected in the sedated and non-sedated groups before (1), during (2), and after (3) endoscopy. Circles = non-sedated group; squares = sedated group. 
upper gastrointestinal endoscopy $(12,17)$. In the present study, serum cortisol, glucose and CRP levels increased during endoscopy, although the increments were not statistically significant. In addition, comparison of the groups with and without sedation revealed that the increments in serum cortisol, glucose and CRP levels were similar in both groups and that the values of the parameters obtained prior to and $10 \mathrm{~min}$ and $1 \mathrm{~h}$ after endoscopy were not statistically different between groups. These results agree with those reported by Yazawa et al. (18), and suggest the view that sedation is not effective in reducing the endocrine response occurring during upper gastrointestinal endoscopy.

Hypoxia developing during upper gastrointestinal endoscopy is a well-recognized complication; however, its cause has not been well clarified. Sedation might primarily cause hypoxia by inducing respiratory depression, while the presence of an endoscope may also cause hypoxia by narrowing the upper airway (19). In a study conducted on 330 patients, Banks et al. (20) demonstrated that the arterial oxygen pressure was considerably decreased in patients with sedation compared to those without, while Wang et al. (19) observed hypoxia in both patients with and without sedation, but stressed that sedation significantly increased hypoxia. Yazawa et al. (18) found a reduction in arterial oxygen pressure in the group with sedation compared to that without, but the reduction was not statistically significant. In another study, Kýlýc et al. (21) reported that sedation with midazolam does not have unfavorable effects on arterial oxygen saturation, but might show unfavorable effects in patients who smoke or patients who have pulmonary problems. In the present study, the patients in both groups were healthy individuals with ASA scores of 1 or 2. Unlike Kýlýc et al. (21), we found significant reductions in $\mathrm{SpO}_{2}$ levels in the sedated group, which was composed of healthy indi- viduals. We think that it would be important to study groups of healthy individuals with ASA scores of 1 or 2 to explore the effects of sedation on cardiopulmonary function while performing upper gastrointestinal endoscopy so the effect of pulmonary diseases on the results of the study was prevented.

Arterial oxygen pressure was reduced in both the groups with and without sedation during endoscopy, with the reduction being statistically significant in the group with sedation, but not in the non-sedated group. However, comparison of the simultaneous arterial oxygen pressures in the sedated and non-sedated groups revealed no statistically significant difference. This situation supports the view that both gastrointestinal endoscopy and sedation might be effective in reducing $\mathrm{SpO}_{2}$ levels.

Ristikankare et al. (22) pointed out that tachycardia plays a more important role than hypoxia in the pathogenesis of cardiorespiratory complications that may develop during upper gastrointestinal endoscopy, and sedation with midazolam causes reduced $\mathrm{SpO}_{2}$ levels, but also prevents the increase in systolic blood pressure and tachycardia observed during endoscopy. Our findings are similar to those of Ristikankare et al. (22) in that we found reductions in $\mathrm{SpO}_{2}$ levels in the group sedated with midazolam. However, we did not observe the favorable influences of sedation on systolic blood pressure and tachycardia observed by these investigators. The absence of a favorable effect of sedation on these two parameters suggests that, because these parameters increase as a result of the endocrine stress response to the endoscope, and because sedation is ineffective against the stress response mediators, it is also ineffective against tachycardia and increased systolic blood pressure.

It is a fact that sedation increases patient tolerance and reduces anxiety in clinical practice, but it should be considered that sedation does not reduce tachycardia and myocardial ischemia and can decrease $\mathrm{SpO}_{2}$ levels. 
In conclusion, we propose that sedation performed with midazolam does not reduce the endocrine stress developing against en- doscopy, but causes a partial increment in the hypoxia that may develop during endoscopy.

\section{References}

1. Hart R, Classen M. Complications of diagnostic gastrointestinal endoscopy. Endoscopy 1990; 22: 229-233.

2. Schenck J, Muller $\mathrm{CH}$, Lubbers $\mathrm{H}$, Mahlke R, Lehnick D, Lankisch PG. Does gastroscopy induce myocardial ischemia in patients with coronary heart disease? Endoscopy 2000; 32: 373-376.

3. Wilcox CM, Faibicher M, Wenger NK, Shalek KA. Prevalence of silent myocardial ischemia and arrhythmias in patients with coronary heart disease undergoing gastrointestinal tract endoscopic procedures. Arch Intern Med 1993; 153: 2325-2330.

4. Cohen LB, Wecsler JS, Gaetano JN, Benson AA, Miller KM, Durkalski V, et al. Endoscopic sedation in the United States: results from a nationwide survey. Am J Gastroenterol 2006; 101: 967-974.

5. Lazzaroni M, Bianchi PG. Preparation, premedication, and surveillance. Endoscopy 2005; 37: 101-109.

6. Leslie K, Stonell CA. Anaesthesia and sedation for gastrointestinal endoscopy. Curr Opin Anaesthesiol 2005; 18: 431-436.

7. Chak A, Rothstein RI. Sedationless upper endoscopy. Rev Gastroenterol Disord 2006; 6: 13-21.

8. Froehlich F, Schwizer W, Thorens J, Kohler M, Gonvers JJ, Fried M. Conscious sedation for gastroscopy: patient tolerance and cardiorespiratory parameters. Gastroenterology 1995; 108: 697-704.

9. Abraham NS, Fallone CA, Mayrand S, Huang J, Wieczorek P Barkun AN. Sedation versus no sedation in the performance of diagnostic upper gastrointestinal endoscopy: a Canadian randomized controlled cost-outcome study. Am J Gastroenterol 2004; 99: 1692-1699.

10. Kohl BA, Deutschman CS. The inflammatory response to surgery and trauma. Curr Opin Crit Care 2006; 12: 325-332.

11. Kehlet $\mathrm{H}$. Manipulation of the metabolic response in clinical practice. World J Surg 2000; 24: 690-695

12. Tonnesen H, Puggaard L, Braagaard J, Ovesen H, Rasmussen V, Rosenberg J. Stress response to endoscopy. Scand J Gastroenterol 1999; 34: 629-631.

13. Lynn RB. Mechanisms of esophageal pain. Am J Med 1992; 92: 11S-19S.
14. Adachi W, Yazawa K, Owa M, Koide N, Hanazaki K, Kajikawa S, et al. Quantification of cardiac stress during EGD without sedation. Gastrointest Endosc 2002; 55: 58-64.

15. Holm C, Rosenberg J. Pulse oximetry and supplemental oxygen during gastrointestinal endoscopy: a critical review. Endoscopy 1996; 28: 703-711.

16. Rosenberg J, Overgaard H, Andersen M, Rasmussen V, Schulze S. Double blind randomised controlled trial of effect of metoprolol on myocardial ischaemia during endoscopic cholangiopancreatography. BMJ 1996; 313: 258-261.

17. Oei-Lim VL, Kalkman CJ, Bartelsman JF, Res JC, van Wezel HB. Cardiovascular responses, arterial oxygen saturation and plasma catecholamine concentration during upper gastrointestinal endoscopy using conscious sedation with midazolam or propofol. Eur $J$ Anaesthesiol 1998; 15: 535-543.

18. Yazawa K, Adachi W, Owa M, Koide N, Hanazaki K, Kajikawa S, et al. Can sedation reduce the cardiac stress during gastrointestinal endoscopy? A study with non-invasive automated cardiac flow measurement by color Doppler echocardiography. Scand J Gastroenterol 2002; 37: 602-607.

19. Wang CY, Ling LC, Cardosa MS, Wong AK, Wong NW. Hypoxia during upper gastrointestinal endoscopy with and without sedation and the effect of pre-oxygenation on oxygen saturation. Anaesthesia 2000; 55: 654-658.

20. Banks MR, Kumar PJ, Mulcahy HE. Pulse oximetry saturation levels during routine unsedated diagnostic upper gastrointestinal endoscopy. Scand J Gastroenterol 2001; 36: 105-109.

21. Kýlýc $M$, Bayan $K$, Yýlmaz $S$, Tüzün $Y$, Dursun $M$, Canoruc $F$. Changes in pulse oximetry levels and factors affecting oxygen saturation during upper gastrointestinal endoscopy with or without sedation. Turk J Gastroenterol 2006; 17: 279-282.

22. Ristikankare $M$, Julkunen $R$, Heikkinen $M$, Mattila $M$, Laitinen $T$, Wang SX, et al. Sedation, topical pharyngeal anesthesia and cardiorespiratory safety during gastroscopy. J Clin Gastroenterol 2006; 40: 899-905. 